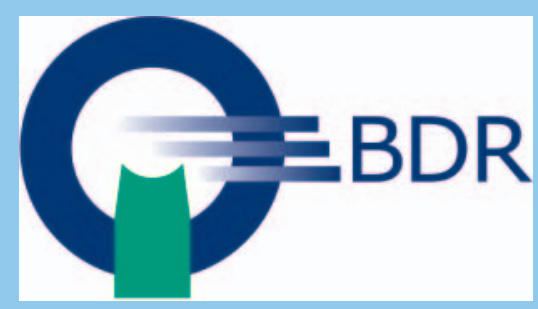

\title{
CIRSE 2018 - Meet - Share - Connect
}

Die Jahrestagung der Cardiovascular and Interventional Radiological Society of Europe (CIRSE) fand in diesem Jahr vom 22.-26. September zum wiederholten Mal in Lissabon statt. Angesichts der Fülle internationaler Teilnehmer aus aller Welt bieten nur wenige Städte Europas neben einem geeigneten Kongresszentrum auch die notwendige Infrastruktur an Hotels und öffentlichem Transportsystem. Auch wenn Letzteres durch einen Taxistreik in diesem Jahr deutlich beeinträchtigt war, bot die Stadt am Tejo dennoch eine beeindruckende Kulisse für das größte Treffen interventionell tätiger Ärzte weltweit. Wie in den vergangenen Jahren fand zeit- und ortsgleich das Interdisciplinary Endovascular Aortic Symposium (IDEAS) in seiner vierten Auflage statt. Das Format im Rahmen des CIRSE-Programms bietet Chirurgen und Interventionalisten die Möglichkeit eines interdisziplinären Austausches zur Therapie von Aorten-Erkrankungen auf höchstem Niveau.

Längst stellt die CIRSE-Tagung den umfassendsten Überblick zu sämtlichen interventionellen Therapieverfahren dar. Dabei können im Wesentlichen 4 Gruppen unterschieden werden: Eingriffe an den arteriellen und venösen Gefäßen, verschließende und ablative Verfahren in der onkologischen Therapie, Versorgung von Aortenaneurysmen sowie die Behandlung primär benigner Erkrankungen mit interventionellen Verfahren. Zu Letzteren zählen ablative Verfahren bei Skelett-Erkrankungen ebenso wie Embolisationen oder fokussierte Ultraschallbehandlung von Uterusmyomen und die Embolisationstherapie beim traumatisierten Patienten. Auch wenn all die genannten Themengruppen in Spezialkongressen wie der European Conference on Interventional Oncology $(\mathrm{ECIO})$ und der European Conference on Embolotherapy (ET) noch eingehender und ausschließlich

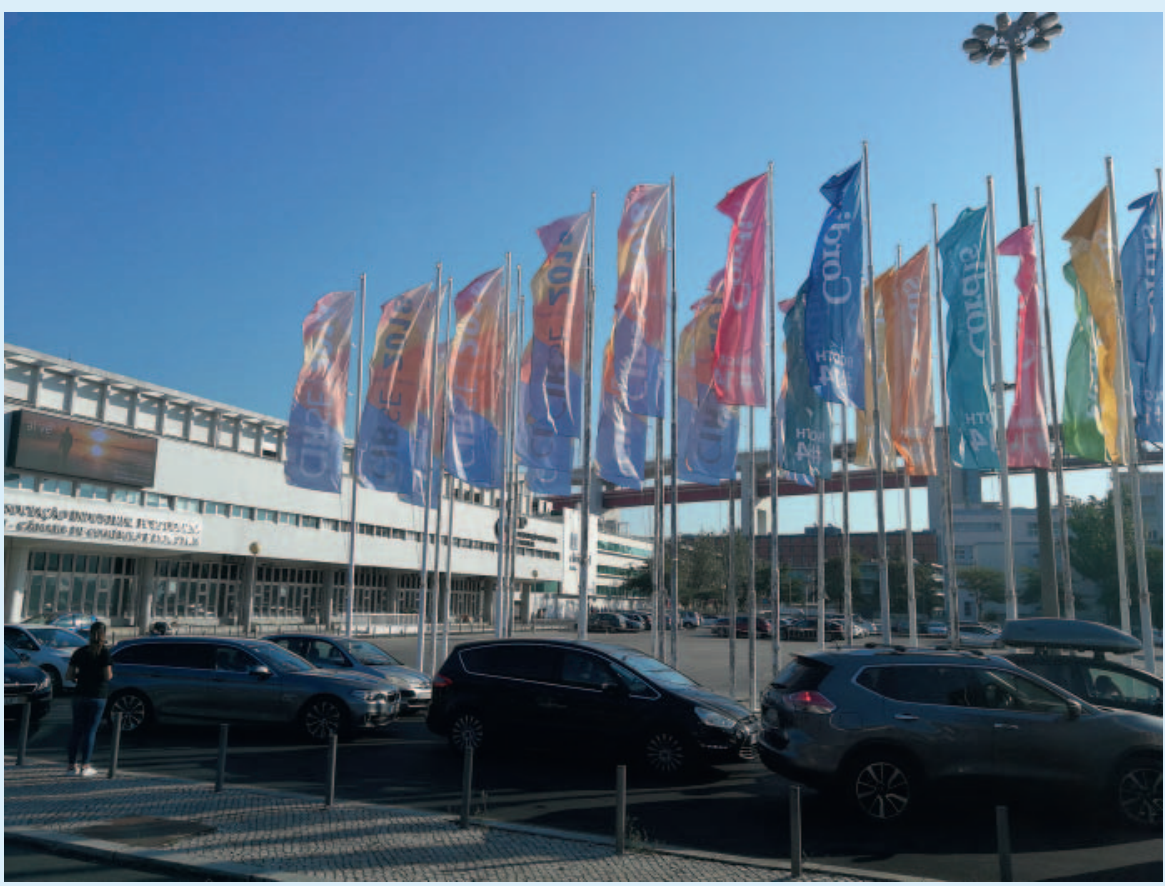

Uber statt Taxi! Wegen des mehrtägigen Taxistreiks war die Improvisation der Kongressteilnehmer gefragt. Foto: BDR

behandelt werden, bietet die CIRSE-Tagung einmal im Jahr die Möglichkeit den Gesamtüberblick zu wahren. Exemplarisch seien einige Ergebnisse aus den einzelnen Gebieten herausgegriffen.

Bei der Re-Kanalisation peripherer arterieller Gefäße wird seit Jahren über die Bedeutung ergänzender Stabilisationsmaßnahmen zur reinen PTA diskutiert. Für den Oberschenkel haben sich Medikamenten-beschichtete Ballone (DEB) weitgehend durchgesetzt. Eine Vielzahl von Studienergebnissen mit unterschiedlich langen Nachbeobachtungsintervallen wurden hierzu vorgestellt. Während bisher vor allem Produkte eines Herstellers gegen andere Verfahren getestet wurden (z. B. DEB der Firma XY gegen die reine PTA) kamen in diesem Jahr erstmals Studien mit dem direkten Vergleich eines vergleichbaren
Produkts unterschiedlicher Hersteller zur Präsentation (z. B. Medikamenten-beschichtete Stents der Firmen Cook und Boston Scientific). Randomisierte Studien dieses Formats sind in den kommenden Jahren in den unterschiedlichsten Bereichen der Interventionellen Radiologie geplant und stellen einen echten Paradigmenwechsel im Studiendesign dar.

Dank der besonderen Beachtung, den die Prostata in jüngster Zeit in der MRT- und PET-CT-Diagnostik erfährt, haben auch Embolisationsverfahren an Bedeutung gewonnen. Bei technischer Machbarkeit muss ihr Stellenwert im Gesamtkonzept aus Operation, Bestrahlung, antihormoneller Therapie und Spezialverfahren wie der PSMA-Therapie allerdings erst evaluiert werden. Eine Renaissance hat die interventionelle Therapie von 


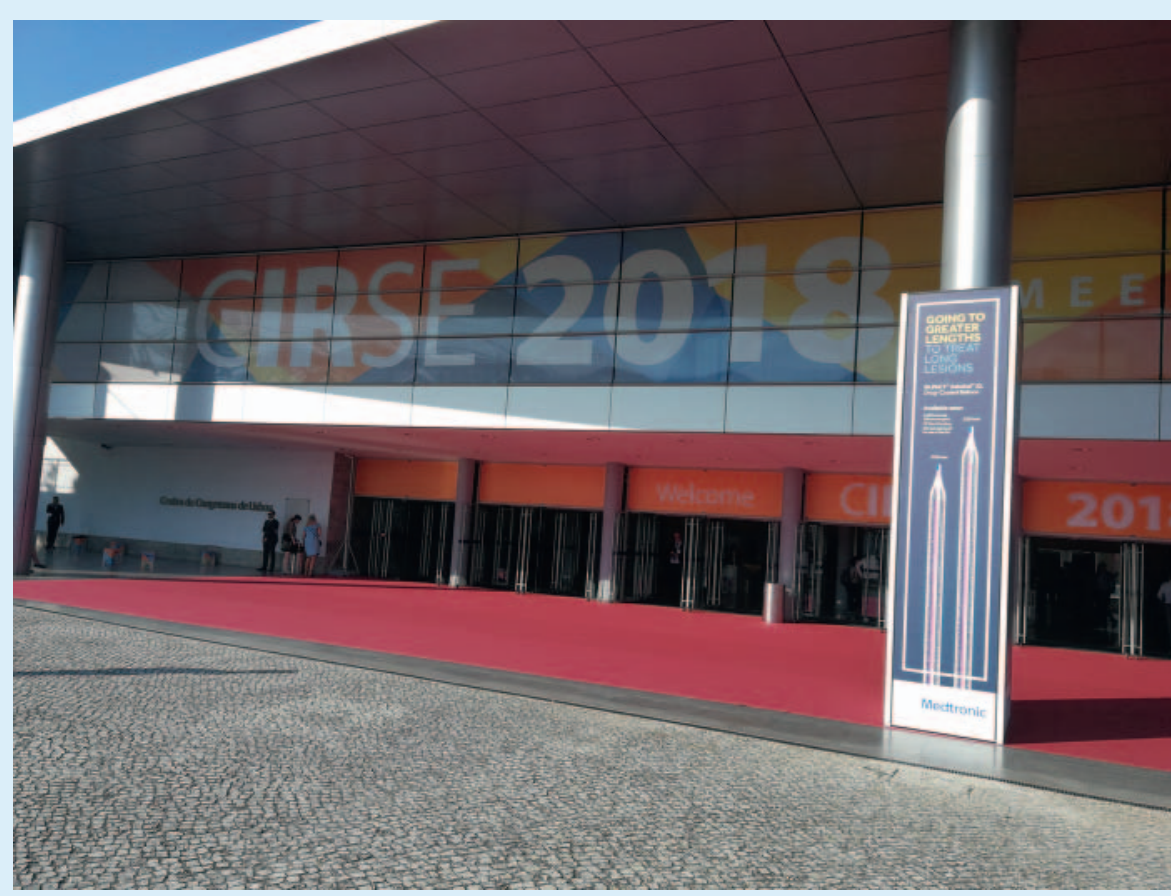

Willkommen zum CIRSE in Lissabon! Foto: BDR

Nierentumoren gefunden. Obwohl unterschiedlichste ablative Verfahren eingesetzt werden, scheint die Kryotherapie die vielversprechendsten Ansätze zu zeigen. Nachdem das Verfahren zwischenzeitlich auch unter den urologischen Operateuren bei kleinen Tumoren favorisiert wird, ist von einer zunehmenden Verbreitung und Anforderung auszugehen. Unstrittig ist inzwischen, auch unter Urologen, dass vor der Therapie eine perkutane histologische Sicherung zwingend erfolgen muss.

Seit dem Beginn der Katheter-gestützten Implantation von Aorten-Prothesen (EVAR) wird über die notwendige Länge des Halses für die proximale Landungszone diskutiert. Erfahrungen aus großen Serien zeigen jetzt, dass potenziell bei einer Vielzahl der Patienten der Prothesenhals für eine klassische EVAR zu kurz ist und deutlich bessere Ergebnisse mit der Vermeidung von Endoleaks durch die frühzeitige Planung einer fenestrierten Prothese erreicht werden können. Die heute zur Verfügung stehenden Materialien und ein Zugang von proximal erlauben es, mit der Indikationsstellung zu dieser Prozedurform großzügiger als bisher umzugehen.

Natürlich wurden auch in diesem Jahr zahlreiche Weiterentwicklungen und Neuvorstellungen in der Industrieausstellung prä- sentiert. Zumeist handelte es sich um Verbesserungen und Fortentwicklungen bereits im Markt etablierter Devices (z. B. größere Ballonlängen bei DEBs, Kombinationen von DEBs und zugehörigen Stents, Verbesserungen der perkutanen Implantation von Dialysefisten). Neu zur Vorstellung kam aber beispielsweise auch ein Ballon-expandierbarer beschichteter Stent-Graft in Größen vor allem für den Beckenbereich.

Beim diesjährigen CIRSE-Kongress wurde auch das 40-jährige Jubiläum der Zeitschrift Cardio Vascular and Interventional Radiology (CVIR) gefeiert. Als exklusives Organ der Gesellschaft steht es allen Mitgliedern zur Verfügung und gehört seit vielen Jahren zu den am höchsten gerankten Journalen der Interventionellen Radiologie. Die Bedeutung der CIRSE als wissenschaftliche Gesellschaft wird auch in der Organisation multinationaler Registerstudien, unter anderem zur SIRT und zur Mikrowellenablation von Lebermetastasen kolorektaler Tumoren, deutlich.

Bereits seit mehreren Jahren sensibilisiert die CIRSE die Anwender unter dem Stichwort „How to make your angio suite smart and safe" für den Strahlenschutz in der Interventionellen Radiologie. Hierzu gibt es Informationen und Veranstaltungen im Radiation-Protection-Pavilion. Nicht erst seit
Inkrafttreten der neuen europäischen Direktiven zum Strahlenschutz ist die Strahlenintensität einzelner interventioneller Verfahren bekannt. Dabei geht es weniger um das reine Einhalten der Vorgaben, die auch in der neuen deutschen Strahlenschutzverordnung zum 01.01.2019 ihren Niederschlag gefunden haben, sondern vor allem um den Schutz der Anwender, die häufig über viele Jahre und mit hohen Untersuchungszahlen der Strahlenbelastung ausgesetzt sind. Eine gute Möglichkeit der Strahlenreduktion wurde dabei für die interventionelle Implantation von AortenProthesen vorgestellt. Durch die exakte Planung und Markierung von zu sondierenden Gefäßabgängen auf einer präoperativen CT kann die Sondierungszeit, damit die Durchleuchtungszeit und folglich die Strahlenbelastung für Operateur und Patient entscheidend gesenkt werden.

Auch wenn man in der eigenen Abteilung wohl kaum das gesamte präsentierte Spektrum der interventionellen Therapie anbieten kann, erlaubt der Besuch des CIRSEJahreskongresses, sich Anregungen und Bestätigung im eigenen Wirkungsspektrum ebenso zu holen, wie Ideen für neue Therapieformen am eigenen Haus zu entwickeln. Einmal mehr hat der diesjährige Kongress gezeigt, dass der interventionell tätige Radiologe eine wichtige Rolle im interdisziplinären Konzert mit Gefäßchirurgen, Angiologen und Onkologen spielt. Angesichts der Herausforderungen, die auf die Radiologie unter dem Stichwort „Künstliche Intelligenz" primär im diagnostischen Bereich zukommen, kann die Bedeutung der Interventionellen Radiologie zum Fortbestand unseres Faches nicht hoch genug eingeschätzt werden. Um diese Rolle auszufüllen, muss man sich, gemäß dem Kongressmotto, treffen, austauschen und vernetzen. Unsere Präsenz in der CIRSE als größte internationale Vertretung ist daher wichtiger denn je.

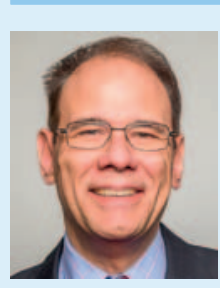

Prof. Dr. Hermann Helmberger, München 